\title{
Thick-brane cosmology
}

\author{
Aqeel Ahmed, ${ }^{a, b, 1}$ Bohdan Grzadkowski $^{a}$ and Jose Wudka ${ }^{b}$ \\ ${ }^{a}$ Faculty of Physics, University of Warsaw, \\ Hoża 69, 00-681 Warsaw, Poland \\ ${ }^{b}$ Department of Physics and Astronomy, UC Riverside, \\ Riverside, CA 92521, U.S.A. \\ E-mail: aqeel.ahmed@fuw.edu.pl, bohdan.grzadkowski@fuw.edu.pl, \\ jose. wudka@ucr.edu
}

ABSTRACT: We search for time-dependent solutions for the 5-dimensional system of a scalar field canonically coupled to gravity. Time-independent and time-dependent scalar field configurations with the most general homogeneous and isotropic $4 \mathrm{D}$ metric are considered. For the case of time-independent scalar field, the time evolution of the scale factor is obtained for different values of the spatial curvature $k=0, \pm 1$. In the case of time-dependent scalar field, two classes of solutions are discussed and an extension of the superpotential formalism is proposed.

Keywords: Large Extra Dimensions, Cosmology of Theories beyond the SM, Classical Theories of Gravity, Field Theories in Higher Dimensions

ArXiv EPRINT: 1312.3576

\footnotetext{
${ }^{1}$ On leave of absence from National Centre for Physics, Quaid-i-Azam University Campus, 45320 Islamabad, Pakistan
} 


\section{Contents}

1 Introduction 1

2 Thick brane cosmological solutions 2

2.1 Static thick brane solutions 3

2.1.1 Evolution of the scale factor 3

2.1.2 Extra-dimensional profiles 6

2.2 Time-dependent thick brane solutions 9

2.2.1 Boosted solutions 9

$\begin{array}{ll}2.2 .2 \text { Twisted solutions } & 10\end{array}$

$\begin{array}{lll}2.2 .3 & \text { Superpotential method } & 12\end{array}$

3 Conclusions 14

A 5D dilaton-like solutions $\quad 15$

\section{Introduction}

The idea of extra space-time dimensions has received a lot of attention since the last decade or so as a possible solution to the hierarchy problem [1-3]. The celebrated proposal by Randall and Sundrum [3], involves one extra-dimension with non-trivial warp factor appearing due to the assumed anti-de Sitter (AdS) geometry along the fifth-dimension. This is the Randall-Sundrum model with two D3-branes on the $S_{1} / Z_{2}$ orbifold along the extra-dimension, which we refer to as RS1. In this model the solution to the hierarchy problem is achieved by the virtue of the non-trivial warped geometry along the extradimension. It was also pointed out by Randall and Sundrum that the extra-dimension can be infinite and yet it can lead to nearly standard $4 \mathrm{D}$ gravity [4]; we refer to this model as RS2, as is customary. The main idea in RS2 is that a single D3-brane of positive tension is embedded in a five-dimensional (5D) AdS geometry and the gravity is effectively fourdimensional (4D) at large distances. The cosmological implications of the extra-dimensions, and in particular to that of RS models, have been studied in detail by many groups [5-13] that found that nearly $4 \mathrm{D}$ cosmological evolutions of our universe is recovered.

All the above-mentioned studies in brane cosmology were done in the presence of "singular branes". The purpose of this paper is to study the cosmology of models where singular branes are replaced by regularized counterparts generated by a 5D scalar field $\phi$ with a non-trivial profile whose effects approach those of a singular brane in an appropriate limit. There have been only few studies in this direction, where the smooth/thick brane cosmological implications have been discussed [14-16]. In this work we consider a scalar field with and without time-dependence in the presence of 5D gravity. For the time-independent 
scalar field configurations, the evolution of the scale factor are discussed and determined. The time-dependent scalar field $\phi(t, y)$ case is discussed with certain assumptions on the metric and an analogue of the traditional [17] superpotential method is developed. It is found that for the case of time-dependent scalar field $\phi(t, y)$ there exists a class of solutions that depend on time $t$ and the 5D coordinate $y$ only through the combination $\eta=c t+d y$, where $c, d$ are constants.

The paper is organized as follows. Section 1 presents an introduction and our motivations. Our results are contained in section 2 , where we separate the case of time independent (subsection 2.1) and time-dependent (subsection 2.2) scalar field profile. Conclusions are collected in section 3 while the appendix A is dedicated to $5 \mathrm{D}$ dilaton-like solutions.

\section{Thick brane cosmological solutions}

We will consider 5D space-times for which the metric takes the following (4D conformal) form,

$$
d s^{2}=a^{2}(t, y) g_{\mu \nu} d x^{\mu} d x^{\nu}+d y^{2},
$$

where $x^{\mu}$ are $4 \mathrm{D}$ coordinates while $g_{\mu \nu}$ is the $4 \mathrm{D}$ metric that we take as the usual RobertsonWalker metric. The function $a(t, y)$ is a scale factor; we will also refer to it as a warp factor because of its $y{ }^{1}$

The action for scalar field in the presence of $5 \mathrm{D}$ gravity is,

$$
S=\int d x^{5} \sqrt{-g}\left\{2 M_{*}^{3} R-\frac{1}{2} g^{M N} \nabla_{M} \phi \nabla_{N} \phi-V(\phi)\right\}
$$

where $M_{*}$ is the Planck mass of the fundamental $5 \mathrm{D}$ theory and $R$ is the $5 \mathrm{D}$ Ricci scalar. We assume that the scalar field $\phi$ depends exclusively on time and the extra coordinate $y$; $V(\phi)$ is the potential for the scalar field.

The Einstein equation and the equation of motion for $\phi$ resulting from the above action (2.2) are

$$
\begin{aligned}
R_{M N}-\frac{1}{2} g_{M N} R & =\frac{1}{4 M_{*}^{3}} T_{M N}, \\
\nabla^{2} \phi-\frac{d V}{d \phi} & =0,
\end{aligned}
$$

where $\nabla^{2}$ is $5 \mathrm{D}$ covariant d'Alembertian operator and the energy-momentum tensor $T_{M N}$ for the scalar field $\phi(t, y)$ is,

$$
T_{M N}=\nabla_{M} \phi \nabla_{N} \phi-g_{M N}\left(\frac{1}{2}(\nabla \phi)^{2}+V(\phi)\right) .
$$

\footnotetext{
${ }^{1}$ In our convention capital Roman indices will refer to $5 \mathrm{D}$ objects, i.e., $M, N, \cdots=0,1,2,3,5$ and the Greek indices label 4D objects, i.e., $\mu, \nu, \cdots=0,1,2,3$, whereas, the lowercase Roman indices $i, j, \cdots=1,2,3$ represent the $3 \mathrm{D}$ spacial coordinates.
} 
The explicit form of the components of the Einstein equation for the metric ansatz (2.1) can be written as, ${ }^{2}$

$$
\begin{array}{ll}
00: & 3\left[\frac{1}{a^{2}} \frac{\dot{a}^{2}}{a^{2}}-\left(\frac{a^{\prime \prime}}{a}+\frac{a^{\prime 2}}{a^{2}}\right)+\frac{k}{a^{2}}\right]=\frac{1}{2} \phi^{\prime 2}+\frac{1}{2} \frac{1}{a^{2}} \dot{\phi}^{2}+V(\phi), \\
i j: & \frac{1}{a^{2}}\left(2 \frac{\ddot{a}}{a}-\frac{\dot{a}^{2}}{a^{2}}\right)-3\left(\frac{a^{\prime \prime}}{a}+\frac{a^{\prime 2}}{a^{2}}\right)+\frac{k}{a^{2}}=\frac{1}{2} \phi^{\prime 2}-\frac{1}{2} \frac{1}{a^{2}} \dot{\phi}^{2}+V(\phi), \\
05: & \frac{a^{\prime}}{a} \frac{\dot{a}}{a}-\frac{\dot{a}^{\prime}}{a}=\frac{1}{3} \phi^{\prime} \dot{\phi}, \\
55: & 3\left[2 \frac{a^{2}}{a^{2}}-\frac{1}{a^{2}} \frac{\ddot{a}}{a}-\frac{k}{a^{2}}\right]=\frac{1}{2} \phi^{\prime 2}+\frac{1}{2} \frac{1}{a^{2}} \dot{\phi}^{2}-V(\phi) .
\end{array}
$$

where $k=0, \pm 1$ denotes the spatial curvature of the $4 \mathrm{D}$ homogeneous and isotropic space-time for Minkowski, de Sitter and anti-de Sitter space, respectively.

The scalar field equation of motion can be written as,

$$
\phi^{\prime \prime}-\frac{1}{a^{2}} \ddot{\phi}+4 \frac{a^{\prime}}{a} \phi^{\prime}-\frac{2}{a^{2}} \frac{\dot{a}}{a} \dot{\phi}-\frac{d V}{d \phi}=0 .
$$

In our notation a prime (dot) denotes a $y(t)$ derivative.

In the following two subsections we will consider two cases, one with time-independent (static) scalar field and the other with time-dependent scalar field.

\subsection{Static thick brane solutions}

In this subsection we will consider a static scalar field scenario, in other words we assume $\phi(t, y)=\phi(y)$, but still allow $a$ to be time-dependent. In this case the Einstein equations (2.6)-(2.9) simplify as follows,

$$
\begin{array}{ll}
00: & 3\left[\frac{1}{a^{2}} \frac{\dot{a}^{2}}{a^{2}}-\left(\frac{a^{\prime \prime}}{a}+\frac{a^{\prime 2}}{a^{2}}\right)+\frac{k}{a^{2}}\right]=\frac{1}{2} \phi^{\prime 2}+V(\phi), \\
i j: & \frac{1}{a^{2}}\left(2 \frac{\ddot{a}}{a}-\frac{\dot{a}^{2}}{a^{2}}\right)-3\left(\frac{a^{\prime \prime}}{a}+\frac{a^{\prime 2}}{a^{2}}\right)+\frac{k}{a^{2}}=\frac{1}{2} \phi^{\prime 2}+V(\phi), \\
05: & \frac{a^{\prime}}{a} \frac{\dot{a}}{a}-\frac{\dot{a}^{\prime}}{a}=0, \\
55: & 3\left[2 \frac{a^{\prime 2}}{a^{2}}-\frac{1}{a^{2}} \frac{\ddot{a}}{a}-\frac{k}{a^{2}}\right]=\frac{1}{2} \phi^{\prime 2}-V(\phi) .
\end{array}
$$

The equation of motion of the scalar field (2.4) has the following form,

$$
\phi^{\prime \prime}+4 \frac{a^{\prime}}{a} \phi^{\prime}-\frac{d V}{d \phi}=0 .
$$

\subsubsection{Evolution of the scale factor}

The assumption that scalar field $\phi$ is time independent implies that $T_{05}=0$, consequentially $G_{05}=0$. This requires $\partial_{t} \partial_{y} \ln a=0$, which implies that $a$ is separable: $a(t, y)=\hat{a}(t) \bar{a}(y)$.

\footnotetext{
${ }^{2}$ For simplicity from here on we will consider the unit system such that $4 M_{*}^{3}=1$.
} 
Using this the remaining Einstein equations become

$$
\begin{array}{ll}
00: & \frac{1}{\hat{a}^{2}} \frac{\dot{\hat{a}}^{2}}{\hat{a}^{2}}+\frac{k}{\hat{a}^{2}}=\frac{\bar{a}^{2}}{3}\left[3\left(\frac{\bar{a}^{\prime \prime}}{\bar{a}}+\frac{\bar{a}^{\prime 2}}{\bar{a}^{2}}\right)+\frac{1}{2} \phi^{\prime 2}+V(\phi)\right], \\
i j: & \frac{1}{\hat{a}^{2}}\left(2 \frac{\ddot{\hat{a}}}{\hat{a}}-\frac{\dot{\hat{a}}^{2}}{\hat{a}^{2}}\right)+\frac{k}{\hat{a}^{2}}=\bar{a}^{2}\left[3\left(\frac{\bar{a}^{\prime \prime}}{\bar{a}}+\frac{\bar{a}^{\prime 2}}{\bar{a}^{2}}\right)+\frac{1}{2} \phi^{\prime 2}+V(\phi)\right], \\
55: & \frac{1}{\hat{a}^{2}} \frac{\ddot{\hat{a}}}{\hat{a}}+\frac{k}{\hat{a}^{2}}=\frac{\bar{a}^{2}}{3}\left[6 \frac{\bar{a}^{\prime 2}}{\bar{a}^{2}}-\frac{1}{2} \phi^{\prime 2}+V(\phi)\right] .
\end{array}
$$

where the left(right) hand sides depend only on $t(y)$. We then obtain the following set of equations for $\hat{a}(t)$ :

$$
\begin{array}{ll}
00: & \frac{1}{\hat{a}^{2}} \frac{\dot{\hat{a}}^{2}}{\hat{a}^{2}}+\frac{k}{\hat{a}^{2}}=C_{t}, \\
i j: & \frac{1}{\hat{a}^{2}}\left(2 \frac{\ddot{\hat{a}}}{\hat{a}}-\frac{\dot{\hat{a}}^{2}}{\hat{a}^{2}}\right)+\frac{k}{\hat{a}^{2}}=C_{x}, \\
55: & \frac{1}{\hat{a}^{2}} \frac{\ddot{\hat{a}}}{\hat{a}}+\frac{k}{\hat{a}^{2}}=C_{y},
\end{array}
$$

where $C_{t, x, y}$ are constants. It is easy to see that in order for the first two equations to be consistent with the third one it is necessary that

$$
C_{y}=\frac{C_{t}+C_{x}}{2} .
$$

On the other hand, form the right hand sides of (2.16)-(2.18) one obtains for the $y$ dependent functions the following equations

$$
\begin{aligned}
00: & C_{t}=\frac{\bar{a}^{2}}{3}\left[3\left(\frac{\bar{a}^{\prime \prime}}{\bar{a}}+\frac{\bar{a}^{2}}{\bar{a}^{2}}\right)+\frac{1}{2} \phi^{2}+V(\phi)\right], \\
i j: & C_{x}=\bar{a}^{2}\left[3\left(\frac{\bar{a}^{\prime \prime}}{\bar{a}}+\frac{\bar{a}^{2}}{\bar{a}^{2}}\right)+\frac{1}{2} \phi^{\prime 2}+V(\phi)\right], \\
55: & C_{y}=\frac{\bar{a}^{2}}{3}\left[6 \frac{\bar{a}^{\prime 2}}{\bar{a}^{2}}-\frac{1}{2} \phi^{\prime 2}+V(\phi)\right] .
\end{aligned}
$$

Then (2.23)-(2.24) immediately imply that

$$
C_{x}=3 C_{t}
$$

so that all the constants can be expressed in terms $C_{y}$ that will be denoted by $\bar{\Lambda}$ :

$$
C_{y}=\bar{\Lambda}, \quad C_{t}=\frac{1}{2} \bar{\Lambda}, \quad C_{x}=\frac{3}{2} \bar{\Lambda} .
$$

Then one finds the following equations that must be satisfied by $\hat{a}(t)$ :

$$
\begin{gathered}
\frac{\ddot{\hat{a}}}{\hat{a}}-2 \frac{\dot{\hat{a}}^{2}}{\hat{a}^{2}}-k=0, \\
\frac{\dot{\hat{a}}^{2}}{\hat{a}^{2}}-\frac{\bar{\Lambda}}{2} \hat{a}^{2}+k=0 .
\end{gathered}
$$



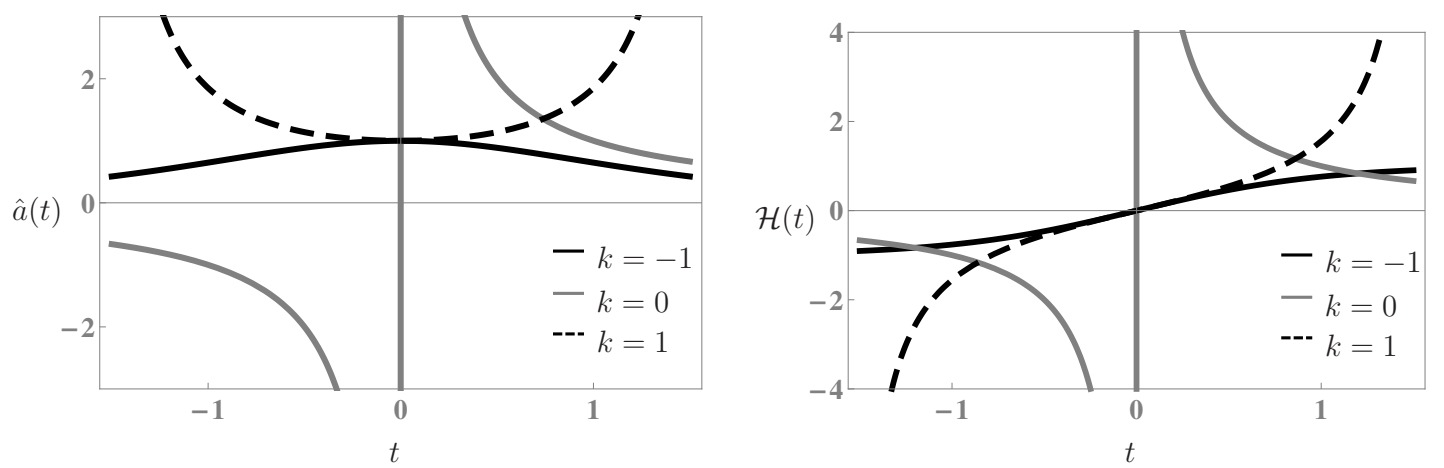

Figure 1. The left graph shows the behavior of the $4 \mathrm{D}$ conformal scale factor $\hat{a}(t)$ as a function of $t$, whereas, the right graph presents the Hubble parameter $\mathcal{H}(t)$ as a function of $t$ for different values of spacial curvature $k$. We choose the value of constants $\alpha_{0}=0$ and $\alpha_{1}=\sqrt{2}$.

As it will be shown below, even though the above equations look independent, solutions that satisfy both of them do exist for each possible $k=0, \pm 1$. Before we proceed, it is worth discussing the relations between (2.28) and (2.29). First note that eq. (2.29) can be rewritten as

$$
\frac{\dot{\hat{a}}^{2}}{\hat{a}^{4}}-\frac{\bar{\Lambda}}{2}+\frac{k}{\hat{a}^{2}}=0,
$$

which is a first integral of eq. (2.28): a derivative of (2.30) reproduces eq. (2.28). So, one can recognize in (2.28) and (2.29) the analogs of classical equation of motion and energy conservation, respectively. Therefore the role of eq. (2.29) is just to adjust "velocity" at the initial moment such that "energy" is properly matched.

Note also that (2.29) is identical with the standard Friedman equation written in terms of the conformal time, thus one finds that $\bar{\Lambda}$ has the interpretation of $4 \mathrm{D}$ cosmological constant.

The solutions of eq. (2.28) are

$$
\hat{a}(t)=|2 / \bar{\Lambda}|^{1 / 2}\left\{\begin{array}{ccc}
\operatorname{sech}\left(t+\alpha_{0}\right) & k=-1 & (\bar{\Lambda}<0) \\
1 /\left(t+\alpha_{0}\right) & k=0 & (\bar{\Lambda}>0) \\
\sec \left(t+\alpha_{0}\right) & k=+1 & (\bar{\Lambda}>0)
\end{array}\right.
$$

where $\alpha_{0}$ is an integration constant. Note that for $k=0,1$ the metric is singular at a finite time $t_{\text {sing }}=-\alpha_{0}+(n+1 / 2) \pi k$, where $n$ is an integer. Using (2.31) it is easy to determine the evolution of the $4 \mathrm{D}$ conformal Hubble parameter $\mathcal{H} \equiv \dot{\hat{a}}(t) / \hat{a}(t)$

$$
\mathcal{H}(t)=\left\{\begin{array}{ccc}
\tanh \left(t+\alpha_{0}\right) & k=-1 & (\bar{\Lambda}<0) \\
1 /\left(t+\alpha_{0}\right) & k=0 & (\bar{\Lambda}>0) \\
\tan \left(t+\alpha_{0}\right) & k=+1 & (\bar{\Lambda}>0)
\end{array} .\right.
$$

Note that when $k=-1, \bar{\Lambda}$ must be negative, which corresponds to anti-de Sitter geometry; for $k=0,+1, \bar{\Lambda}$ must be positive, thus representing de Sitter space-time. In figure 1 we have plotted the scale factor $\hat{a}(t)$ and the Hubble parameter $\mathcal{H}(t)$ for $k=0, \pm 1$. 
When $\bar{\Lambda}=0$ there are no (real) solutions when $k=+1$. When $k=-1$, known as the Milne universe in the conventional cosmology, eqs. (2.24)-(2.25) reduce to the standard static equations considered e.g. in $[17,18]$. In this case time-dependent part of the scale factor is determined by eqs. (2.28)-(2.29), whose general solution is a linear combination of the following functions

$$
\hat{a}_{ \pm}(t)=\alpha_{ \pm} e^{ \pm \sqrt{-k} t} \quad k=-1,0
$$

Note that the static solution requires $k=0$ and $\bar{\Lambda}=0$.

In the following section we focus on the $y$-dependent solutions of eqs. (2.24) and (2.25).

\subsubsection{Extra-dimensional profiles}

In this section we will determine $y$-dependent part of solutions that are governed by eqs. (2.24)-(2.25). For this purpose it is useful to define $\bar{a}(y) \equiv e^{A(y)}$, such that our $y$-dependent Einstein equations eqs. (2.24)-(2.25) and the scalar field equation (2.15) can be written as,

$$
\begin{aligned}
3 A^{\prime \prime}+\frac{3}{2} \bar{\Lambda} e^{-2 A} & =-\phi^{2}, \\
6 A^{\prime 2}-3 \bar{\Lambda} e^{-2 A} & =\frac{1}{2} \phi^{\prime 2}-V(\phi), \\
\phi^{\prime \prime}+4 A^{\prime} \phi^{\prime}-\frac{d V}{d \phi} & =0 .
\end{aligned}
$$

The procedure we follows begins by assuming $A(y)$ is a known function, so the above conditions are to be considered as equations to determine $\phi(y)$ and $V(\phi) .^{3}$

Specifically, we will consider the following form of the warp function $A(y)$,

$$
A(y)=-\ln \cosh (\beta y)
$$

where $\beta$ is a parameter. The above choice is dictated by simplicity and by a desire to have a warp factor which behaves as $\sim \exp (-|y|)$ at large $y$, so that it mimics RS solutions and the hierarchy problem can be in principle approached. This choice of $A(y)$ approximates well the static solution obtained e.g. in $[17,18]$ for a kink profile of the scalar field. Figure 2 shows the warp function and its derivatives.

It is difficult to find an exact analytical solution for $\phi(y)$ from eq. (2.34), however if one considers separately regions of small $\left(|y| \lesssim \beta^{-1}\right)$ and large $\left(|y| \gtrsim \beta^{-1}\right) y$ then approximate analytical solutions are easy to obtain. When $\beta$ is large (which is the case of our interest) then for small values of $y$, one can ignore ${ }^{4}$ the exponential term in eq. (2.34), i.e.

$$
3 A^{\prime \prime}=-\phi^{\prime 2}, \quad(y \rightarrow 0)
$$

\footnotetext{
${ }^{3}$ In the literature there are few known analytic de Sitter and anti-de Sitter solutions of the system (2.34)(2.35), see for example [19-23].

${ }^{4}$ When $y \rightarrow 0$ then $A(y) \rightarrow 0$ and $A^{\prime \prime} \rightarrow-\beta^{2}$, therefore in the vicinity of $y=0$ the eq. (2.34) behaves as $-3 \beta^{2}+\frac{3}{2} \bar{\Lambda}=-\phi^{\prime 2}$, implying $\phi_{s}(y)$ linear in $y$. For values of $\beta$ adopted here the $\bar{\Lambda}$ term is negligible.
} 


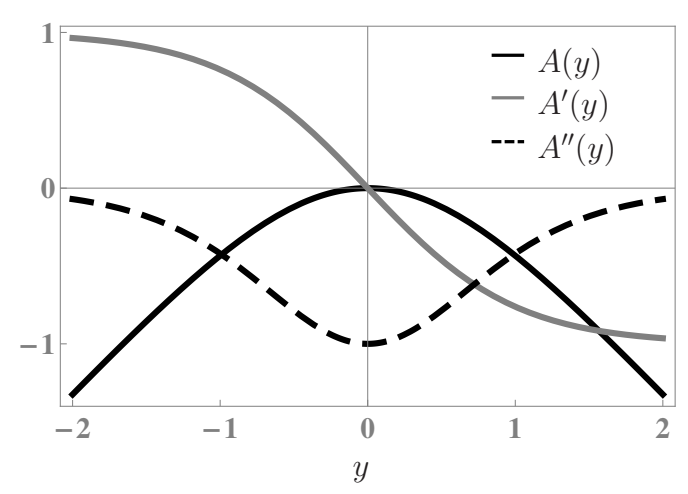

Figure 2. The warp function $A(y)$ and its derivatives $A^{\prime}(y)$ and $A^{\prime \prime}(y)$ as a function of $y$ for $\beta=1$.

with the following solution

$$
\phi_{s}(y)=2 \sqrt{3} \arctan [\tanh (\beta y / 2)], \quad(y \rightarrow 0)
$$

where $\phi_{s}(y)$ denotes the solution for small $y$. It is important to note that dropping the exponential term in eq. (2.34) is a reasonable assumption in the vicinity of $y=0$ if $\beta$ is larger than $\bar{\Lambda}$, as illustrated in figure 3 for $\beta=5$ and $\bar{\Lambda}=-1$. On the other hand, for large values of $y$, the exponential term dominates in eq. (2.34) and we can ignore $A^{\prime \prime}(y)$, i.e.,

$$
\frac{3}{2} \bar{\Lambda} e^{-2 A}=-\phi^{\prime 2}
$$

with $\bar{\Lambda}<0$. The solution of above equation reads

$$
\phi_{l}(y)=\sqrt{-\frac{3 \bar{\Lambda}}{2}} \frac{1}{\beta} \sinh (\beta y), \quad(|y| \rightarrow \infty)
$$

where $\phi_{l}(y)$ denotes the solution valid for large values of $y$.

In figure 3 we have plotted the approximate analytic solutions $\phi_{s, l}(y)$ and the exact numerical one $\phi_{n}(y)$. For large $y$ the quality of the approximation can be easily estimated from the figure; one finds that for $|y| \gtrsim 5 \beta^{-1}, \phi_{n} \simeq \phi_{l}$. For small $y$ the right panel of the figure shows that for $|y| \lesssim \beta^{-1}, \phi_{n} \simeq \phi_{s}$. In the intermediate region $\beta^{-1} \lesssim|y| \lesssim 5 \beta^{-1}$ the approximations $\phi_{n, s}$ are less accurate. It is also worth to mention that as $\beta$ grows the region of applicability of $\phi_{s}$ shrinks, and $\phi_{l}$ converges to the exact numerical solution $\phi_{n}$.

There is a comment here in order. If, instead of (2.37), we had used the solution obtained in the static case of [18], then for $\bar{\Lambda}=0$ we would reproduce exactly the kink profile for the scalar field and the corresponding potential as in [18]. In that case (with $k=-1)$, the time evolution of the scale factor would be governed by (2.33) while the scalar profile would preserve its shape. In this special case the time evolution in $4 \mathrm{D}$ and source (the scalar field profile) along the extra dimension fully decouple, so that the scalar profile is retained while non-trivial time evolution of the scale factor has purely $4 \mathrm{D}$ nature.

We then substitute the solutions we obtained for $A(y)$ and $\phi(y)$ in (2.35) to obtain the scalar potential $V(\phi)$, which we plot as a function of $\phi$ in figure 4 . To get approximate 

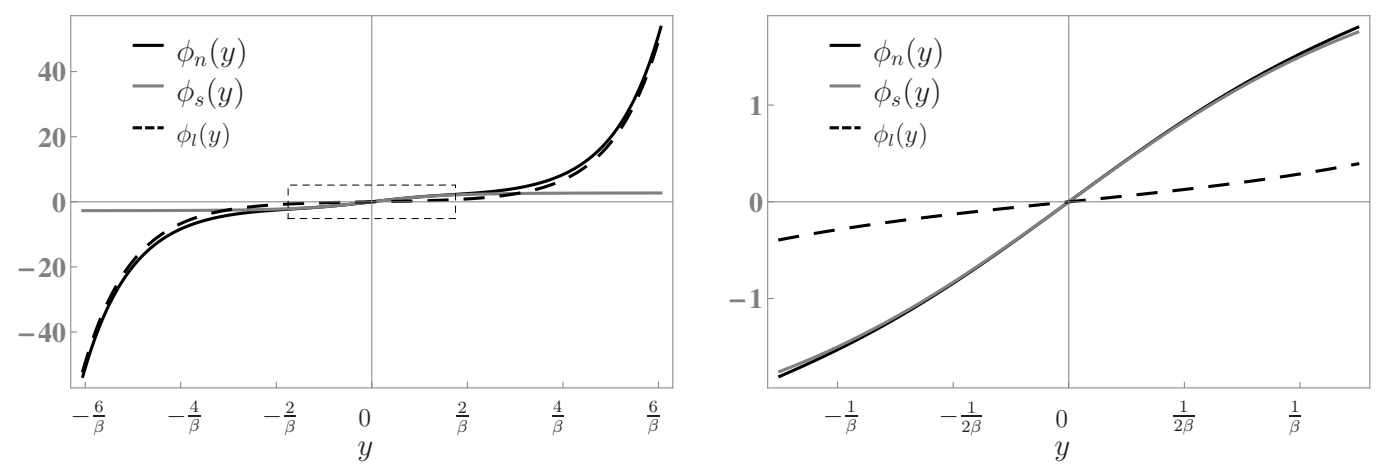

Figure 3. These graphs show the exact numerical solution for the scalar field $\phi_{n}(y)$, the approximate analytic solution for small $\left(\phi_{s}(y)\right)$ and large $\left(\phi_{l}(y)\right)$ values of $y$ as a function of $y$ in units of $\beta^{-1}$ for $\beta=5$ and $\bar{\Lambda}=-1$. The right graph shows the zoomed central region of the left graph.

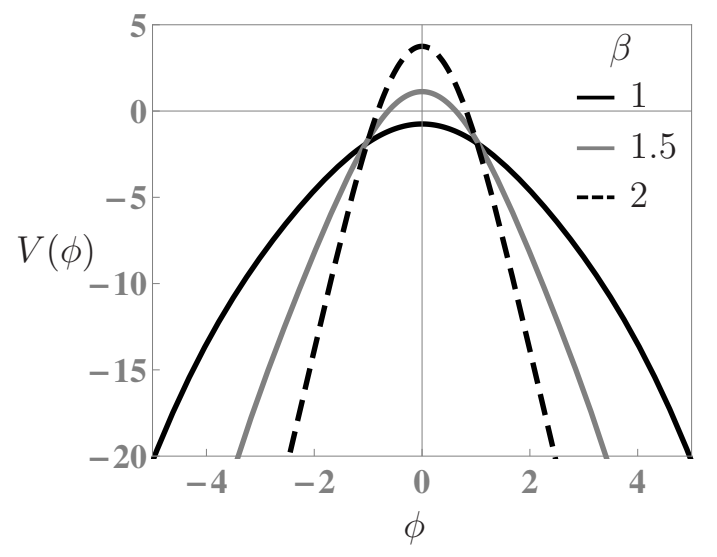

Figure 4. The scalar potential $V(\phi)$ as a function of $\phi$ for different values of $\beta$ and $\bar{\Lambda}=-1$.

analytic results for the scalar potential $V(\phi)$ corresponding to small and large values of $\phi(y)$, we use the Einstein equation (2.35) along with the analytic solutions of scalar field $\phi_{s}(y)$ and $\phi_{l}(y)$. For small values of scalar field $\phi_{s}(y)$ the scalar potential is, ${ }^{5}$

$$
V_{s}(\phi)=\left(\frac{3}{2} \beta^{2}+\frac{9}{4} \bar{\Lambda}\right)+\left(-\frac{5}{2} \beta^{2}+\frac{3}{4} \bar{\Lambda}\right) \phi^{2}+\mathcal{O}\left(\phi^{4}\right), \quad(\phi \rightarrow 0) .
$$

For large values of scalar field $\phi_{l}(y)$ we can write the potential as,

$$
V_{l}(\phi)=-\frac{3}{2} \beta^{2} \phi^{2}+\left(-6 \beta^{2}+\frac{9}{4} \bar{\Lambda}\right)+\mathcal{O}\left(\phi^{-2}\right), \quad(|\phi| \rightarrow \infty) .
$$

Note that the potential is unbounded from below. In fact this is a generic consequence of the requirement that the warp function $A(y)$ has a linear dependence on $y$ as $|y| \rightarrow \infty$. Indeed, as seen from (2.34)-(2.35), for large $y, V(y) \sim \frac{9}{4} \bar{\Lambda} e^{-2 A} \sim \frac{9}{4} \bar{\Lambda} e^{2 \beta|y|}$. Since at large $|y|, \phi \propto e^{\beta|y|}$ we find $V(\phi) \propto-\phi^{2}$, so the potential is always unbounded from below.

\footnotetext{
${ }^{5}$ For $\bar{\Lambda}=0$ one would reproduce the standard bottom of a wine bottle potential as in [18].
} 
An alternative approach to solve eqs. (2.34)-(2.35) is to reduce these equations into the following first order equations by the use of an extension of the superpotential method [17],

$$
\begin{aligned}
A^{\prime} & =-\frac{1}{3} \mathcal{W} \gamma(y), \\
\phi^{\prime} & =\frac{1}{\gamma(y)} \frac{\partial \mathcal{W}}{\partial \phi}, \\
V & =\frac{1}{\gamma(y)^{2}}\left(\frac{\partial \mathcal{W}}{\partial \phi}\right)^{2}-\frac{2}{3} \mathcal{W}^{2},
\end{aligned}
$$

where $\gamma(y)$ is defined by,

$$
\gamma(y)=\left(1+\frac{9}{2} \frac{\bar{\Lambda}}{\mathcal{W}^{2}} e^{-2 A(y)}\right)^{1 / 2} .
$$

The superpotential method reduces the second order non-linear differential equations (2.34)(2.36) to the system of first order nonlinear differential equations (2.44)-(2.46), though obtaining the solution are less straightforward than when $\bar{\Lambda}=0$ (for $\bar{\Lambda}=0$ see, for example, $[17,18])$. Unfortunately, for the present case, one can not start with a desired shape for the scalar profile, our strategy is instead to solve a system of first order nonlinear equations by first choosing the warp function $A(y)$, next solve eq. (2.44) algebraically for $\mathcal{W}(y)$, and then solve the following equation for $\phi(y)$

$$
\phi^{\prime}(y)=\sqrt{\frac{\mathcal{W}^{\prime}(y)}{\gamma(y)}} .
$$

Then eq. (2.46) gives the potential $V(y)$ which can be written as $V(\phi)$ after inverting $\phi(y)$ to $y(\phi)$. We will not further investigate these solutions for $\phi(y)$, we only note that choosing $A(y)$ as in (2.37) one would reproduce the result obtained earlier in this subsection.

\subsection{Time-dependent thick brane solutions}

In this subsection we will look for solutions of the Einstein equations (2.6)-(2.9) allowing for time-dependence of the scalar field.

\subsubsection{Boosted solutions}

In this subsection we show how the a static solution for the warp factor, $a(y)$ and scalar field $\phi(y)$ can be promoted to a time-dependent solution through a boost along the extra dimension: $y \rightarrow y^{\prime}=\gamma(v t+y)$, where $\gamma=1 / \sqrt{1-v^{2}}$ and $v$ is a relative velocity. It proves to be more convenient first to redefine the fifth coordinate $y$ so that the length element (2.1) is written as

$$
d s^{2}=a^{2}(z)\left(\eta_{\mu \nu} d x^{\mu} d x^{\nu}+d z^{2}\right),
$$

Let us consider a Lorentz transformation $t^{\prime}=\gamma(t+v z)$ and $z^{\prime}=\gamma(v t+z)$. It is easy to check that $a^{\prime}\left(t^{\prime}, z^{\prime}\right)=a(t, z)$, since $\phi$ is a scalar field $\phi^{\prime}\left(t^{\prime}, z^{\prime}\right)=\phi(t, z)$. By general covariance $a^{\prime}\left(t^{\prime}, z^{\prime}\right)$ and $\phi^{\prime}\left(t^{\prime}, z^{\prime}\right)$ are also solutions on the Einstein equations. Therefore we conclude that for any given stationary solution $a(y)$ and $\phi(y)$, the functions $a[\gamma(-v t+z(y))]$ and $\phi[\gamma(-v t+z(y))]$ also satisfy the Einstein equations. This strategy could be applied to any stationary solution, e.g. to the kink solution discussed in [18]. 


\subsubsection{Twisted solutions}

In this subsection we return to the fifth dimensional coordinate $y$. We will to show that one can obtain a class of interesting solutions assuming that $a$ and $\phi$ depend on $y$ and $t$ only through the combination $\eta \equiv c t+d y^{6}$ where $c$ and $d$ are non-zero constants. In the next subsection we will show that if the superpotential method is used, the 05 component of the Einstein equations in fact implies such a dependence on $\eta$ for $\phi$. With this assumption the Einstein equations (2.6)-(2.9) become,

$$
\begin{array}{ll}
00: \quad & 3 \frac{c^{2}}{a^{2}} \frac{a^{\prime 2}}{a^{2}}-3 d^{2}\left(\frac{a^{\prime \prime}}{a}+\frac{a^{\prime 2}}{a^{2}}\right)+\frac{3 k}{a^{2}}=\frac{d^{2}}{2} \phi^{\prime 2}+\frac{1}{2} \frac{c^{2}}{a^{2}} \phi^{\prime 2}+V(\phi), \\
i j: & \frac{c^{2}}{a^{2}}\left(2 \frac{a^{\prime \prime}}{a}-\frac{a^{\prime 2}}{a^{2}}\right)-3 d^{2}\left(\frac{a^{\prime \prime}}{a}+\frac{a^{\prime 2}}{a^{2}}\right)+\frac{k}{a^{2}}=\frac{d^{2}}{2} \phi^{\prime 2}-\frac{1}{2} \frac{c^{2}}{a^{2}} \phi^{\prime 2}+V(\phi), \\
05: & \frac{a^{\prime \prime}}{a}-\frac{a^{\prime 2}}{a^{2}}=-\frac{1}{3} \phi^{\prime 2}, \\
55: & 6 d^{2} \frac{a^{\prime 2}}{a^{2}}-3 \frac{c^{2}}{a^{2}} \frac{a^{\prime \prime}}{a}-\frac{3 k}{a^{2}}=\frac{d^{2}}{2} \phi^{\prime 2}+\frac{1}{2} \frac{c^{2}}{a^{2}} \phi^{\prime 2}-V(\phi) .
\end{array}
$$

Where now a prime denotes an $\eta$ derivative. If now we add eqs. (2.51)-(2.53), and then use of eq. (2.52) we obtain

$$
\frac{a^{\prime \prime}}{a}+\frac{a^{\prime 2}}{a^{2}}+\frac{2 k}{c^{2}}=0
$$

On the other hand, subtracting eqs. (2.50)-(2.51) and using (2.54) gives

$$
\frac{a^{\prime 2}}{a^{2}}+\frac{k}{c^{2}}=\frac{1}{6} \phi^{\prime 2} .
$$

Using the above two relations in eq. (2.53), we obtain the following form for the scalar potential $V(\phi)$

$$
V(\phi)=d^{2}\left(-\frac{1}{2} \phi^{\prime 2}+\frac{6 k}{c^{2}}\right)
$$

At this point the strategy is clear, one first solves $(2.54)$ for $a(\eta)$, then $\phi$ is easily determined from (2.55). If $\phi(\eta)$ is an invertible function of $\eta$ then $V(\phi)$ can be found from (2.56). In the following we will find such solutions for each possible value of $k$.

$\boldsymbol{k}=\mathbf{0}$. In this case the warp factor $a(\eta)$ is obtained by integrating eq. (2.54):

$$
a(\eta)=a_{0}\left(1+2 b_{0} \eta\right)^{1 / 2},
$$

where $a_{0}$ and $b_{0}$ are integration constants. It is important to note that the above solution is only valid in the region of the space-time where $\eta>-1 / 2 b_{0}$, and we will see below that the scalar field is singular at $\eta \rightarrow-1 / 2 b_{0}$. With this explicit expression for $a(\eta)$, we use eq. (2.55) to find the scalar field $\phi(\eta)$ :

$$
\phi(\eta)= \pm \sqrt{\frac{3}{2}} \ln \left(1+2 b_{0} \eta\right)+\phi_{0},
$$

\footnotetext{
${ }^{6}$ Note that this is not a boost of a stationary solution.
} 


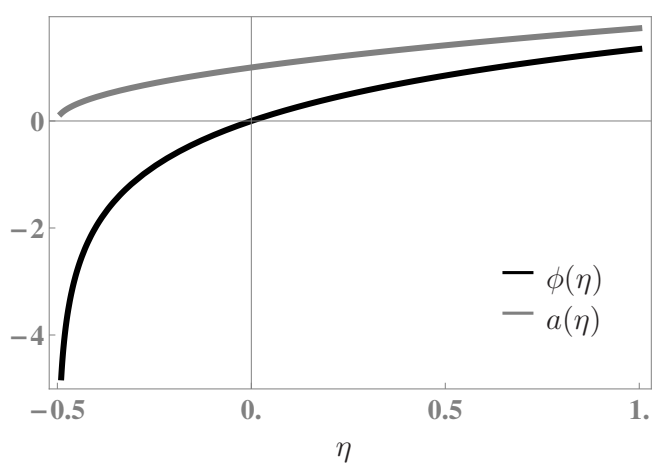

Figure 5. This plot shows the behavior of $\phi(\eta)(2.58)$ and $a(\eta)(2.57)$ as a function of $\eta$ for $k=0$, $a_{0}=b_{0}=1$ and $\phi_{0}=0$.

where $\phi_{0}$ is an integration constant. Then the scalar potential takes the form

$$
V(\phi)=-3 b_{0}^{2} e^{-\sqrt{\frac{8}{3}}\left(\phi-\phi_{0}\right)} .
$$

It is noteworthy that the above potential is similar to the dilaton potential $V_{\text {dilaton }}=$ $-|\Lambda| e^{\sqrt{4 / 3} \phi}$ studied in a 5D context e.g. in [24]; in our case, however, the argument of the exponent is $\sqrt{8 / 3} \phi$, while in [24] it is $\sqrt{(4 / 3)} \phi$. In the appendix A we discuss this issue in details.

Figure 5 shows the behavior of the scalar field $\phi(\eta)$ and the warp factor $a(\eta)$.

$\boldsymbol{k}=\mathbf{1}$. In this case the warp factor $a(\eta)$ found from (2.54) reads

$$
a(\eta)=a_{0} \sqrt{\cos \left(2 \eta / c+c_{0}\right)},
$$

where $a_{0}$ and $c_{0}$ are integration constants. The above solution is applicable in the region $\left|\eta+c c_{0} / 2\right|<c \pi / 4$. With this expression for $a(\eta)$, we use eq. (2.55) to find the scalar field $\phi(\eta):$

$$
\phi(\eta)=\sqrt{\frac{3}{2}} \ln \left(\frac{1+\tan \left(\eta / c+c_{0} / 2\right)}{1-\tan \left(\eta / c+c_{0} / 2\right)}\right)+\phi_{0},
$$

where $\phi_{0}$ is an integration constant. Then the scalar potential is given by

$$
V(\phi)=\frac{3 d^{2}}{2 c^{2}}\left[3-\cosh \left(\sqrt{\frac{8}{3}}\left(\phi-\phi_{0}\right)\right)\right] .
$$

It is important to note that these solutions for $k=1$ case have a singularity at $\eta=$ $\left( \pm \pi-2 c_{0}\right) c / 4$. Figure 6 illustrates the behavior of the scalar field $\phi(\eta)$ and the warp factor $a(\eta)$.

$\boldsymbol{k}=\mathbf{- 1}$. In this case (2.54) yields

$$
a(\eta)=a_{0} \sqrt{\cosh \left(2 \eta / c+c_{0}\right)},
$$

where $a_{0}$ and $c_{0}$ are integration constants. In this case, however, there are no real solutions for $\phi^{\prime}(\eta)$, so we will not consider this possibility further. 


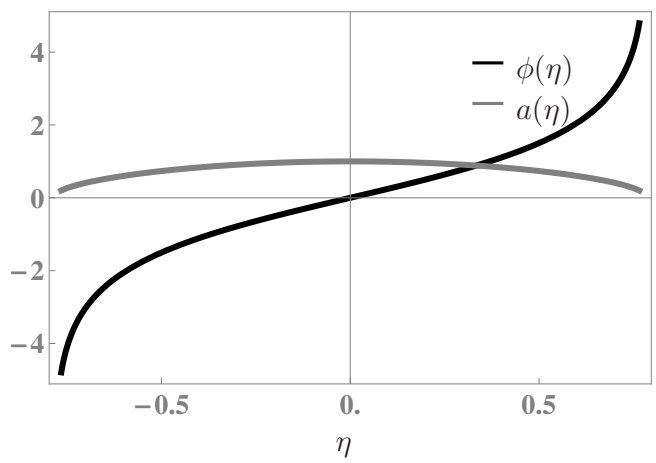

Figure 6. This plot shows the behavior of $\phi(\eta)(2.61)$ and $a(\eta)(2.60)$ as a function of $\eta$ for $k=1$, $a_{0}=c=1$ and $\phi_{0}=c_{0}=0$.

\subsubsection{Superpotential method}

It is instructive to develop an analogue of the superpotential method for the time dependent scalar field in 5D warped space-time. We define the following quantities,

$$
\frac{a^{\prime}}{a} \equiv-\frac{1}{3} W(\phi), \quad \frac{\dot{a}}{a} \equiv-\frac{1}{3} H(\phi),
$$

where $W(\phi)$ and $H(\phi)$ are functions of $\phi(t, y)$. In the above equation and in the following, unless otherwise stated, we return to denote $t$ and $y$ derivatives by a dot and a prime respectively. With the above definitions we find from the 05 component of the Einstein equations (2.8),

$$
\frac{\partial W(\phi)}{\partial \phi}=\phi^{\prime}, \quad \frac{\partial H(\phi)}{\partial \phi}=\dot{\phi} .
$$

Now, if we re-express the 55 (or 00) component of the Einstein equations in terms of the superpotential variables $W(\phi)$ and $H(\phi)$ through eqs. (2.64) and (2.65) we get the potential $V(\phi)$ as,

$$
V(\phi)=\frac{1}{2}\left(\frac{\partial W(\phi)}{\partial \phi}\right)^{2}-\frac{2}{3} W(\phi)^{2}-\frac{1}{a^{2}}\left(\frac{1}{2}\left(\frac{\partial H(\phi)}{\partial \phi}\right)^{2}-\frac{1}{3} H(\phi)^{2}-3 k\right) .
$$

The $i j$ component of the Einstein equation (2.7) then gives

$$
\frac{1}{2}\left(\frac{\partial H(\phi)}{\partial \phi}\right)^{2}-\frac{1}{3} H(\phi)^{2}-3 k=0 .
$$

For the $k=0$ case this gives

$$
\frac{\partial H(\phi)}{\partial \phi} \frac{1}{H(\phi)}= \pm \sqrt{\frac{2}{3}} .
$$

with solution

$$
H(\phi)=H_{0} e^{ \pm \sqrt{\frac{2}{3}} \phi} .
$$


where, $H_{0} \equiv H(0)$ is a constant of integration. ${ }^{7}$ It is important to note that the superpotentials $W(\phi)$ and $H(\phi)$ are related to each other since from (2.65) one obtains

$$
\frac{\partial^{2} W(\phi)}{\partial \phi^{2}} \dot{\phi}=\dot{\phi}^{\prime}, \quad \frac{\partial^{2} H(\phi)}{\partial \phi^{2}} \phi^{\prime}=\dot{\phi}^{\prime},
$$

which implies, along with eq. (2.65), that,

$$
\frac{\partial^{2} W(\phi)}{\partial \phi^{2}} \frac{\partial H(\phi)}{\partial \phi}=\frac{\partial^{2} H(\phi)}{\partial \phi^{2}} \frac{\partial W(\phi)}{\partial \phi} .
$$

Hence,

$$
W(\phi)=A_{0} H(\phi)+W_{0}
$$

where $A_{0}$ and $W_{0}$ are constants on integration.

In order to determine $\phi$ we use (2.67) together with (2.65) to obtain,

$$
\dot{\phi}= \pm \sqrt{\frac{2}{3}} H_{0} e^{ \pm \sqrt{\frac{2}{3}} \phi} .
$$

On the other hand, from (2.65) and (2.72) we find,

$$
\phi^{\prime}= \pm \sqrt{\frac{2}{3}} A_{0} H_{0} e^{ \pm \sqrt{\frac{2}{3}} \phi},
$$

Therefore from eqs. (2.73) and (2.74), we have,

$$
\dot{\phi}(t, y)=\frac{1}{A_{0}} \phi^{\prime}(t, y)
$$

which implies that, as claimed previously, $\phi$ can depend on $t$ and $y$ only through $\eta$ (with $d=A_{0} c$ ). For simplicity, hereafter we choose $c=d=1$. Then from eq. (2.73) we obtain

$$
\frac{d \phi(\eta)}{d \eta}= \pm \sqrt{\frac{2}{3}} H_{0} e^{ \pm \sqrt{\frac{2}{3}} \phi(\eta)}
$$

with solution

$$
\phi(\eta)=\mp \sqrt{\frac{3}{2}} \ln \left(-\frac{2}{3} H_{0} \eta+e^{\mp \sqrt{\frac{2}{3}} \phi_{0}}\right),
$$

where $\phi_{0}$ is an integration constant. Note that the above solution is valid only for $-\frac{2}{3} H_{0} \eta+$ $e^{\sqrt{\frac{2}{3}} \phi_{0}}>0$ and there is a singularity at $-\frac{2}{3} H_{0} \eta+e^{\sqrt{\frac{2}{3}} \phi_{0}}=0$. Also one can see that for the choice $H_{0}=-3 b_{0}$ and $\phi_{0}=0$ the above result for $\phi(\eta)$ matches the one obtained in (2.58).

In order to determine the warp factor $a$ we use eq. (2.64):

$$
\frac{\dot{a}}{a} \equiv-\frac{1}{3} H(\phi)=\mp \sqrt{\frac{1}{6}} \frac{\partial H(\phi)}{\partial \phi}=\mp \sqrt{\frac{1}{6}} \dot{\phi},
$$

\footnotetext{
${ }^{7}$ Notice that $H(\phi)=H_{0} e^{ \pm \sqrt{\frac{2}{3}}|\phi|}$ is also a solution of $(2.67)$ even though the first derivative of $\phi$ is discontinuous which suggest that the solutions will require presence of a singular brane. We will not consider such solution in this study.
} 
which can be solved to obtain $a(t, y)$ as,

$$
a(t, y)=a\left(t_{0}, y\right) e^{\mp \sqrt{\frac{1}{6}}\left(\phi(t, y)-\phi\left(t_{0}, y\right)\right)}
$$

where $a\left(t_{0}, y\right)$ and $\phi\left(t_{0}, y\right)$ are functions of $y$ at the constant time slice $t_{0}$. $a\left(t_{0}, y\right)$ can be found by substituting the above expression for $a(t, y)$ into the first equation in eq. (2.64); we then find

$$
a\left(t_{0}, y\right)=a\left(t_{0}, y_{0}\right) e^{\mp \sqrt{\frac{1}{6}}\left(\phi\left(t_{0}, y\right)-\phi\left(t_{0}, y_{0}\right)\right)-\frac{1}{3} W_{0} y}
$$

inserting this in eq. (2.79) we find

$$
a(t, y)=a\left(t_{0}, y_{0}\right) e^{\mp \sqrt{\frac{1}{6}}\left(\phi(t, y)-\phi\left(t_{0}, y_{0}\right)\right)-\frac{1}{3} W_{0} y}
$$

where $a\left(t_{0}, y_{0}\right)$ and $\phi\left(t_{0}, y_{0}\right)$ are constants. Since $\phi(t, y)=\phi(\eta)$; then, for $\phi\left(t_{0}, y_{0}\right) \equiv \phi_{0}=0$, $W_{0}=0$ and $H_{0}=-3 b_{0}$, we recover the result that $a(t, y) \equiv a(\eta)$ as in eq. (2.57).

$$
a(\eta)=a_{0}\left(1+2 b_{0} \eta\right)^{1 / 2}
$$

Since $W(\phi)$ has been found we can determine the potential $V(\phi)$ directly from (2.66)

$$
V(\phi)=-\frac{1}{3}\left(A_{0} H_{0} e^{ \pm \sqrt{\frac{2}{3}} \phi}+2 W_{0}\right)^{2}+\frac{2}{3} W_{0}^{2}
$$

We recover the result for $V(\phi)$ as in eq. (2.59) with $H_{0}=-3 b_{0}, A_{0}=1, W_{0}=0$ and the lower sign (minus sign) in the exponent.

Similarly one can reproduce all the results obtained in section 2.2 adopting the superpotential method for non-zero $k$ values. Therefore we can conclude that the superpotential method is equivalent to the assumption that $\phi$ and $a$ depend on $t$ and $y$ only through $\eta=c t+d y$. The main advantage the this method this that it reduces the second order differential equations into to first order equations which are much easier to solve analytically.

\section{Conclusions}

In this work we have analyzed a 5D scenario with a scalar field in the presence of gravity. We have found solutions of the Einstein equations for the case of time-independent scalar field assuming a conformal form of the $4 \mathrm{D}$ metric. Both the evolution of the scale factor, its extra-dimensional shape and the profile of scalar field were discussed and determined for different values of spacial curvature $k=0, \pm 1$. Also for the time-dependent scalar field $\phi(t, y)$ and $4 \mathrm{D}$ conformal metric, analytic solutions were obtained in certain cases. We have also formulated a superpotential method for $t$ - and $y$-dependent profiles of the scalar field. For the solution which has been found both the scalar filed $\phi$ and the scale factor $a$ depend on time $t$ and $y$ only through $\eta=c t+d y$, where $c$ and $d$ are constants. 


\section{Acknowledgments}

BG thanks Jacek Pawelczyk for useful remarks. AA and BG are grateful to the NORDITA Program "Beyond the LHC" for hospitality where some part of this work is done. This work has been supported in part by the National Science Centre (Poland) as a research project, decision no DEC-2011/01/B/ST2/00438. AA acknowledges financial support from the Foundation for Polish Science International PhD Projects Programme co-financed by the EU European Regional Development Fund.

\section{A 5D dilaton-like solutions}

Here we are going to consider a stationary setup defined by the action (2.2) with the metric ansatz (2.1) with $a(t, y) \equiv a(y)$ and $\phi(t, y)=\phi(y)$. The resulting Einstein equations and the equation of motion for $\phi$ (with $4 M_{*}^{3}=1$ ) are as follows ${ }^{8}$

$$
\begin{aligned}
& 6 \frac{a^{\prime 2}}{a^{2}}=\frac{1}{2}\left(\phi^{\prime}\right)^{2}-V(\phi), \\
& 3 \frac{a^{\prime \prime}}{a}+3 \frac{a^{\prime 2}}{a^{2}}=-\frac{1}{2}\left(\phi^{\prime}\right)^{2}-V(\phi), \\
& \phi^{\prime \prime}+4 \frac{a^{\prime}}{a} \phi^{\prime}-\frac{d V}{d \phi}=0,
\end{aligned}
$$

where a prime denotes a $y$ derivative. We assume that the scalar potential $V(\phi)$ can be expressed in terms of the superpotential $W(\phi)$ as $[17,18]$,

$$
V(\phi)=\frac{1}{2}\left(\frac{\partial W(\phi)}{\partial \phi}\right)^{2}-\frac{2}{3} W(\phi)^{2}
$$

where $W(\phi)$ satisfies the following relations,

$$
\phi^{\prime}=-\frac{\partial W(\phi)}{\partial \phi} \quad \text { and } \quad \frac{a^{\prime}}{a}=\frac{1}{3} W(\phi) .
$$

In addition we focus on the following form of dilatonic superpotential $W(\phi)$

$$
W(\phi)=W_{0} e^{\frac{\epsilon}{2} \phi},
$$

where $W_{0}$ and $\epsilon$ are constants and as we will see the different values of $\epsilon$ will correspond to a class of different solutions. The resulting scalar potential $V(\phi)$ is,

$$
V(\phi)=W_{0}^{2}\left(\frac{\epsilon^{2}}{8}-\frac{2}{3}\right) e^{\epsilon \phi} .
$$

The scalar field $\phi(y)$ and the warped function $A(y)$ obtained from eq. (A.5) are

$$
\begin{aligned}
& \phi(y)=-\frac{2}{\epsilon} \ln \left(1+\frac{\epsilon^{2}}{4} W_{0} y\right) \\
& a(y)=a_{0}\left(1+\frac{\epsilon^{2}}{4} W_{0} y\right)^{\frac{4}{3 \epsilon^{2}}} .
\end{aligned}
$$

\footnotetext{
${ }^{8}$ In this case $k=0$ is required by the Einstein equations.
} 
The above result (A.7)-(A.8) and (A.9) represents a class of solutions parameterized by $\epsilon$. For $\epsilon= \pm \sqrt{\frac{8}{3}}$, we recover our results found in eqs. (2.57)-(2.58) and (2.59) for $W_{0}=3 b_{0}$. Whereas, for $\epsilon= \pm \sqrt{\frac{4}{3}}$ we recover the linear dilaton solution discussed by Antoniadis et al. [24]. It is instructive to notice that the metric given by eq. (11) of ref. [24] coincides with (A.9) for $\alpha=W_{0}$.

Open Access. This article is distributed under the terms of the Creative Commons Attribution License (CC-BY 4.0), which permits any use, distribution and reproduction in any medium, provided the original author(s) and source are credited.

\section{References}

[1] N. Arkani-Hamed, S. Dimopoulos and G.R. Dvali, The Hierarchy problem and new dimensions at a millimeter, Phys. Lett. B 429 (1998) 263 [hep-ph/9803315] [INSPIRE].

[2] I. Antoniadis, N. Arkani-Hamed, S. Dimopoulos and G.R. Dvali, New dimensions at a millimeter to a Fermi and superstrings at a TeV, Phys. Lett. B 436 (1998) 257 [hep-ph/9804398] [INSPIRE].

[3] L. Randall and R. Sundrum, A Large mass hierarchy from a small extra dimension, Phys. Rev. Lett. 83 (1999) 3370 [hep-ph/9905221] [INSPIRE].

[4] L. Randall and R. Sundrum, An Alternative to compactification, Phys. Rev. Lett. 83 (1999) 4690 [hep-th/9906064] [INSPIRE].

[5] P. Binetruy, C. Deffayet, U. Ellwanger and D. Langlois, Brane cosmological evolution in a bulk with cosmological constant, Phys. Lett. B 477 (2000) 285 [hep-th/9910219] [INSPIRE].

[6] P. Binetruy, C. Deffayet and D. Langlois, Nonconventional cosmology from a brane universe, Nucl. Phys. B 565 (2000) 269 [hep-th/9905012] [INSPIRE].

[7] J.M. Cline, C. Grojean and G. Servant, Cosmological expansion in the presence of extra dimensions, Phys. Rev. Lett. 83 (1999) 4245 [hep-ph/9906523] [INSPIRE].

[8] C. Csáki, M. Graesser, C.F. Kolda and J. Terning, Cosmology of one extra dimension with localized gravity, Phys. Lett. B 462 (1999) 34 [hep-ph/9906513] [INSPIRE].

[9] C. Csáki, M. Graesser, L. Randall and J. Terning, Cosmology of brane models with radion stabilization, Phys. Rev. D 62 (2000) 045015 [hep-ph/9911406] [InSPIRE].

[10] E.E. Flanagan, S.H.H. Tye and I. Wasserman, Cosmological expansion in the Randall-Sundrum brane world scenario, Phys. Rev. D 62 (2000) 044039 [hep-ph/9910498] [INSPIRE].

[11] P. Kanti, I.I. Kogan, K.A. Olive and M. Pospelov, Single brane cosmological solutions with a stable compact extra dimension, Phys. Rev. D 61 (2000) 106004 [hep-ph/9912266] [INSPIRE].

[12] P. Kanti, I.I. Kogan, K.A. Olive and M. Pospelov, Cosmological three-brane solutions, Phys. Lett. B 468 (1999) 31 [hep-ph/9909481] [INSPIRE].

[13] D. Bazeia, F.A. Brito and F.G. Costa, First-order framework and domain-wall/brane-cosmology correspondence, Phys. Lett. B 661 (2008) 179 [arXiv:0707.0680] [INSPIRE]. 
[14] M. Giovannini, Time-dependent gravitating solitons in five dimensional warped space-times, Phys. Rev. D 76 (2007) 124017 [arXiv:0708.1830] [InSPIRE].

[15] D.P. George, M. Trodden and R.R. Volkas, Extra-dimensional cosmology with domain-wall branes, JHEP 02 (2009) 035 [arXiv:0810.3746] [INSPIRE].

[16] A. Kadosh, A. Davidson and E. Pallante, Slinky evolution of domain wall brane cosmology, Phys. Rev. D 86 (2012) 124015 [arXiv:1202.5255] [INSPIRE].

[17] O. DeWolfe, D.Z. Freedman, S.S. Gubser and A. Karch, Modeling the fifth-dimension with scalars and gravity, Phys. Rev. D 62 (2000) 046008 [hep-th/9909134] [INSPIRE].

[18] A. Ahmed and B. Grzadkowski, Brane modeling in warped extra-dimension, JHEP 01 (2013) 177 [arXiv: 1210.6708] [INSPIRE].

[19] M. Gremm, Thick domain walls and singular spaces, Phys. Rev. D 62 (2000) 044017 [hep-th/0002040] [INSPIRE].

[20] S. Kobayashi, K. Koyama and J. Soda, Thick brane worlds and their stability, Phys. Rev. D 65 (2002) 064014 [hep-th/0107025] [INSPIRE].

[21] A. Wang, Thick de Sitter 3 branes, dynamic black holes and localization of gravity, Phys. Rev. D 66 (2002) 024024 [hep-th/0201051] [INSPIRE].

[22] N. Sasakura, A de Sitter thick domain wall solution by elliptic functions, JHEP 02 (2002) 026 [hep-th/0201130] [INSPIRE].

[23] V.I. Afonso, D. Bazeia and L. Losano, First-order formalism for bent brane, Phys. Lett. B 634 (2006) 526 [hep-th/0601069] [INSPIRE].

[24] I. Antoniadis, A. Arvanitaki, S. Dimopoulos and A. Giveon, Phenomenology of TeV Little String Theory from Holography, Phys. Rev. Lett. 108 (2012) 081602 [arXiv:1102.4043] [INSPIRE]. 